\title{
ON EQUIVALENCE OF CERTAIN TYPES OF SERIES OF ORTHONORMAL FUNCTIONS
}

\author{
G. H. PEEBLES
}

If one has a set of "properly" independent functions $u_{1}(x), u_{2}(x), \cdots$, $u_{n}(x), \cdots$ and a function $p(x)$ which on an interval $(a, b)$ is non-negative, integrable and such that $\int_{a}^{b} p(x) d x>0$, one can construct a second set $v_{1}(x), v_{2}(x), \cdots, v_{n}(x), \cdots\left[v_{n}(x)=a_{n 1} u_{1}(x)+\cdots+a_{n n} u_{n}(x)\right.$, where $a_{n k}$ is a constant] whose members satisfy the relations

$$
\int_{a}^{b} p(x) v_{n}(x) v_{m}(x) d x=\delta_{n m} .
$$

Associated with such sets of orthonormal functions is the problem of expanding an "arbitrary" function $f(x)$ in a convergent series of the members of a particular set, that is, the problem of determining conditions sufficient for

$$
\lim _{n \rightarrow \infty} \sum_{k=1}^{n} v_{k}(x) \int_{a}^{b} p(t) f(t) v_{k}(t) d t=f(x) .
$$

One approach to the problem lies in showing that expansion (1) is equivalent to another expansion, usually a Fourier series, whose behavior is known. ${ }^{1}$ In the case of orthogonal polynomials, where $u_{n}(x)=x^{n-1}$, the writer was able to show, under conditions not too restrictive, that the expansions of a function in terms of the two sets of orthogonal polynomials corresponding, respectively, to different weight functions converge to the same value or diverge together. ${ }^{2}$ It is the purpose of this note to point out that similar results can be obtained for systems of orthonormal functions constructed from a set $\left[u_{n}(x)\right]$ which has the property that the product of any two members

Presented to the Society, September 7, 1939; received by the editors August 7, 1941. Results presented to the Society, April 14, 1939 under the title The boundedness of certain systems of orthogonal functions, are implicit in the brief discussion on boundedness of orthonormal functions which appears at the end of this paper.

${ }_{1}^{1}$ Theorems and references on equiconvergence for orthogonal polynomials are given by G. Szegö, Orthogonal Polynomials, American Mathematical Society Colloquium Publications, vol. 23, 1939, chaps. 9 and 13. For equivalence for another type of orthogonal functions, see J. Mercer, Sturm-Liouville series of normal functions in the theory of integral equations, Philosophical Transactions of the Royal Society of London, (A), vol. 211 (1912), pp. 111-198; pp. 174-177.

${ }^{2}$ An equivalence theorem for series of orthogonal polynomials, Proceedings of the National Academy of Sciences, vol. 3 (1939), pp. 97-104. 
$u_{n}(x) u_{m}(x)$ is a linear combination of the first $n+m+\beta$ members, where $\beta$ may depend on $n$ and $m$, but never exceeds some integer, say, $j$. A set with this property, for instance, underlies a set of polynomials in two variables orthogonal on an arc of an algebraic curve. $^{3}$ If the curve is the hyperbola $y^{2}=x^{2}-1$, the set $\left[u_{n}(x)\right]$ is $1, x,\left(x^{2}-1\right)^{1 / 2}, x^{2}, x\left(x^{2}-1\right)^{1 / 2}, \cdots$. The system of trigonometric functions $1, \sin x, \cos x, \sin 2 x, \cos 2 x, \cdots$, which is essentially a set of polynomials orthogonal on a circle, will be used later for the purposes of illustration.

The proof of the equivalence of expansions is usually made to depend on asymptotic formulas for the orthogonal functions. ${ }^{4}$ The method used here, however, rests chiefly on the familiar closure formula of Parseval and a fundamental property of orthonormal functions, namely, that $\int_{a}^{b} p(x)\left[\phi(x)-\sum_{i}^{n} c_{k} v_{k}(x)\right]^{2} d x$ is a minimum with respect to the choice of the values of the $c$ 's when $c_{k}=\int_{a}^{b} p(x) \phi(x) v_{k}(x) d x$. In addition to the assumption that $u_{n}(x) u_{m}(x)$ is a linear combination of not more than the first $n+m+j$ members, it is convenient to suppose at the outset that the $u$ 's and all functions to be introduced are such that Parseval's closure formula holds and the necessary integrals exist whenever the argument so requires.

Let $\left[v_{n}(x)\right]$ and $\left[w_{n}(x)\right]$ be the sets of orthonormal functions constructed from the $u$ 's on the interval $(a, b)$ and associated, respectively, with the weight functions $p(x)$ and $q(x)=r(x) p(x)$. Let

$$
s_{n}(x)=\sum_{i=1}^{n} b_{i} v_{i}(x)=\sum_{i=1}^{n} v_{i}(x) \int_{a}^{b} p(t) f(t) v_{i}(t) d t
$$

and

$$
S_{n}(x)=\sum_{i=1}^{n} w_{i}(x) \int_{a}^{b} q(t) f(t) w_{i}(t) d t
$$

be the corresponding $n$th order partial sums of the expansions of a function $f(x)$. The difference between the two sums is a linear combination of the $u$ 's, and so may be expressed in terms of the $w$ 's:

$$
S_{n}(x)-s_{n}(x)=\sum_{k=1}^{n} r_{k n} w_{k}(x) .
$$

The coefficient

\footnotetext{
${ }^{3}$ See Dunham Jackson, Orthogonal polynomials on a plane curve, Duke Mathematical Journal, vol. 3 (1937), pp. 228-236; pp. 232-235; Orthogonal polynomials in three variables, ibid., vol. 4 (1938), pp. 441-454.

${ }^{4}$ See Footnote 1.
} 


$$
\begin{aligned}
r_{k n} & =\int_{a}^{b} q(x) w_{k}(x)\left[S_{n}(x)-s_{n}(x)\right] d x \\
& =\int_{a}^{b} p(x)\left[r(x) w_{k}(x)\right]\left[f(x)-s_{n}(x)\right] d x .
\end{aligned}
$$

Hence, if one sets $B_{i k}=\int_{a}^{b} p(x) r(x) w_{k}(x) v_{i}(x) d x$, the assumption of the validity of Parseval's formula allows one to write

$$
r_{k n}=\sum_{i=n+1}^{\infty} b_{i} B_{i k}
$$

which with Cauchy's inequality yields the relation

$$
\left|r_{k n}\right| \leqq\left\{\sum_{i=n+1}^{\infty} b_{i}^{2}\right\}^{1 / 2}\left\{\sum_{i=n+1}^{\infty} B_{i k}^{2}\right\}^{1 / 2}
$$

Since $\lim _{n \rightarrow \infty} \sum_{n+1}^{\infty} b_{i}^{2}=0$, it is clear from the last inequality and relation (2) that, if one writes $l_{n k}=\left\{\sum_{n+1}^{\infty} B_{i k}^{2}\right\}^{1 / 2}$, the existence of $L=\lim _{n \rightarrow \infty} \sum_{k=1}^{n} l_{n k}$ is sufficient for

$$
\lim _{n \rightarrow \infty}\left[S_{n}(x)-s_{n}(x)\right]=0
$$

for any value of $x$ for which $\left|w_{k}(x)\right|$ is bounded for all values of $k$.

The preceding result depends on Parseval's formula and not on the rule of combination assumed for the $u$ 's. A more serviceable form stating that $L$ surely exists, if the ratio function $r(x)$ belongs to a suitable class of functions, is obtained when the special rule of combination holds.

The integral formula

$$
l_{n k}^{2}=\sum_{i=n+1}^{\infty} B_{i k}^{2}=\int_{a}^{b} p(x)\left[r(x) w_{k}(x)-\sum_{i=1}^{n} B_{i k} v_{i}(x)\right]^{2} d x
$$

provides a means for getting at conditions sufficient for the existence of $L$. An immediate consequence of (3) is that $l_{n k}$ is bounded for all values of $n$ and $k$, if $r(x)$ is bounded on $(a, b)$; for, if $C$ is an upper bound of $r(x)$, by Bessel's inequality for orthogonal functions,

$$
l_{n k}^{2} \leqq \int_{a}^{b} p(x)\left[r(x) w_{k}(x)\right]^{2} d x=\int_{a}^{b} C r(x) p(x)\left[w_{k}(x)\right]^{2} d x=C .
$$

This result will be needed immediately.

Suppose $C \geqq r(x)$ and $r(x)$ is a linear combination of the $u$ 's of order $m$. Then $r(x) w_{k}(x)$ is a linear combination of the $u$ 's and consequently of the $v$ 's, of order not more than $m+k+j$. The integral of formula (3) 
vanishes for $n \geqq m+k+j$; therefore $l_{n k}=0$ for $k \leqq n-(m+j)$, and $L$ exists since always $\sum_{k=1}^{n} l_{n k} \leqq(m+j) C$.

Suppose that on $(a, b) C \geqq r(x) \geqq c>0$ and that $r(x)=\lim _{n \rightarrow \infty} G_{n}(x)$, where $G_{n}(x)$ is a linear combination of the $u$ 's with $\epsilon_{n}$, the maximum or least upper bound of $\left|r(x)-G_{n}(x)\right|$ on $(a, b)$, such that $\sum_{1}^{\infty} \epsilon_{n}$ is convergent. Since $w_{k}(x) G_{n-k-j}(x)$ is a linear combination of the $v$ 's of order $n$ at most, one has for $k \leqq n-j-1$

$$
\begin{aligned}
l_{n k} & \leqq\left\{\int_{a}^{b} p(x)\left[w_{k}(x) r(x)-w_{k}(x) G_{n-k-j}(x)\right]^{2} d x\right\}^{1 / 2} \\
& \leqq \epsilon_{n-k-j}\left\{\int_{a}^{b} p(x)\left[w_{k}(x)\right]^{2} d x\right\}^{1 / 2} \\
& \leqq \epsilon_{n-k-j}\left\{c^{-1} \int_{a}^{b} p(x) r(x)\left[w_{k}(x)\right]^{2} d x\right\}^{1 / 2}=c^{-1 / 2} \epsilon_{n-k-j}
\end{aligned}
$$

For the remaining $j+1$ values of $k(n-j, n-j+1, \cdots, n), l_{n k} \leqq C^{1 / 2}$. Hence $L$ exists.

The next assumption concerning the nature of $r(x)$ is very specific, but the result, when combined with that obtained in the case where $r(x)$ is a linear combination of the $u$ 's, may increase the generality of the results as an example will show later. On $(a, b)$ suppose, unlike the last case, the lower bound of $r(x)$ is zero, but there exists an $m$ th order linear combination of the $u$ 's $h_{m}(x)$ such that $C \geqq r(x) \geqq\left[h_{m}(x)\right]^{2}$ and such that $r(x) / h_{m}(x)$, like $r(x)$ in the preceding case, can be approximated by a set of $G$ 's. Then for $k \leqq n-m-2 j-1$

$$
\begin{aligned}
l_{n k} & =\left\{\int_{a}^{b} p(x)\left[h_{m}(x) w_{k}(x)\right]^{2}\left[r(x) / h_{m}(x)-G_{n-m-k-2 j}(x)\right]^{2} d x\right\}^{1 / 2} \\
& \leqq \epsilon_{n-m-k-2 j}\left\{\int_{a}^{b} p(x) r(x)\left[w_{k}(x)\right]^{2} d x\right\}^{1 / 2}=\epsilon_{n-m-k-2 j} .
\end{aligned}
$$

As before, if $\sum_{1}^{\infty} \epsilon_{n}$ is convergent, $L$ exists.

A review of the conditions imposed on $r(x)$ discloses that in no case is $r(x)$ allowed to become infinite and that only under very restrictive hypotheses is $r(x)$ allowed to vanish. If $r(x)$ were discontinuous, one would expect, in general, that approximating sums of the sort required would not exist. An example in trigonometric functions on the interval $(-\pi, \pi)$ will indicate briefly how and when the results just obtained may be used to circumvent difficulties presented by such exceptional points.

In the case of trigonometric functions, theorems due to Dunham Jackson provide the desired information about the approximating 
sums. ${ }^{5}$ For present purposes it suffices to know that a function of period $2 \pi$ which possesses everywhere a $k$ th order derivative satisfying a Lipschitz condition of order $\alpha$ can be approximated by a trigonometric sum of order $n$ so closely that the absolute value of the error nowhere exceeds $K / n^{k+\alpha}, K$ a constant. Hence, if $r(x) \geqq c>0$ and has a derivative which satisfies a Lipschitz condition of order $\alpha$ and if $r(x)$ and $f(x)$ are of classes $L_{p}(-\pi, \pi)$ and $L_{p}^{2}(-\pi, \pi)$ so that Parseval's formula holds, one has equivalence between the expansions of $f(x)$ in the two sets of trigonometric sums corresponding to $p(x)$ and $r(x) p(x)$ wherever the sums associated with $r(x) p(x)$ are bounded. Suppose, however, that $r(x)$, a function of period $2 \pi$, is bounded, stays away from zero and has a second derivative everywhere in $(-\pi, \pi)$ except at $x=0$. At the origin, suppose $r(x)$ tends to vanish on the left and to become infinite on the right, but in such a way that $r(x) \geqq \sin ^{2} x$ everywhere and $\lim _{x=0+} \sin x p(x)$ exists. The function described has no particular merit except that it serves to illustrate how exceptional points may be handled. Let it again be assumed that $f(x)$ and $r(x)$ are of classes $L_{p}(-\pi, \pi)$ and $L_{p}^{2}(-\pi, \pi)$. It follows from the first case considered, where $r(x)$ is a linear combination of the $u$ 's, that $L$ corresponding to the weight functions $\sin ^{10} x r(x) p(x)$ and $r(x) p(x)$ exists. On the other hand $\sin ^{10} x r(x) \geqq\left(\sin ^{6} x\right)^{2}$ and $\sin ^{10} x r(x) / \sin ^{6} x$, on removing the discontinuity at the origin, has a second derivative everywhere and so can be approximated by an $n$th order trigonometric sum with the absolute value of the error not exceeding $K / n^{2}$. Hence, by virtue of the third case, $L$ corresponding to the weight functions $\sin ^{10} x r(x) p(x)$ and $p(x)$ also exists. Therefore the expansions of $f(x)$ corresponding to $r(x) p(x)$ and $p(x)$ are each equivalent to the expansion corresponding to $\sin ^{10} x r(x) p(x)$, whereever the sums orthonormal with respect to the last weight function are bounded. It follows that the expansions associated with $r(x) p(x)$ and $p(x)$ are equivalent, wherever the sums orthonormal with respect to $\sin ^{10} x r(x) p(x)$ are bounded.

The results on equivalence given here depend, in part, on the boundedness of the functions orthonormal with respect to $r(x) p(x)$. There is at hand in the foregoing discussion a result which may be useful in this connection. Referring to the definition of $B_{i k}$, one sees that formally

$$
r(x) w_{k}(x)=\sum_{i=k-j}^{\infty} B_{i k} v_{i}(x)
$$

${ }^{5}$ Dunham Jackson, The Theory of Approximation, American Mathematical Society Colloquium Publications, vol. 11, 1930, chap. 1. 
and

$$
v_{i}(x)=\sum_{k=1}^{i} B_{i k} w_{k}(x) .
$$

Since $B_{i k} \leqq\left\{\sum_{j=1}^{\infty} B_{j k}^{2}\right\}^{1 / 2}=l_{i-1, k}$, in all the cases of the ratio function $r(x)$ considered, the right-hand members of (4) and (5) are absolutely convergent and bounded, wherever, respectively, the $v$ 's and $w$ 's are bounded. Hence, if conditions are such that the right-hand member of (4) converges to the value of the left-hand member and if a set of points is known for which the v's are bounded, then the w's are bounded on the same set except where $r(x)=0$. Similarly, boundedness of the w's leads through (5) to results on the boundedness of the $v$ 's.

UnIVERSITY OF Minnesota

\section{A MAPPING CHARACTERIZATION OF PEANO SPACES}

O. G. HARROLD, JR.

The Hahn-Mazurkiewicz theorem states that any Peano space (compact, connected, locally connected, metric space) is a continuous image of the interval $0 \leqq t \leqq 1$, and conversely. Clearly, the mapping function is not uniquely determined. If the Peano space $\mathscr{X}$ has special topological properties, the mapping may be selected in a simpler fashion than might be expected generally. On the other hand, special properties of $\mathcal{X}$ may impose certain necessary restrictions on the mapping. For example, if $\mathcal{X}$ is a regular continuum in the sense of Menger, then, by a theorem due to Nöbeling, ${ }^{1}$ there is a continuous mapping $f$ of the circle ${ }^{2}$ onto $\mathcal{X}$ such that each point of finite order is covered by the mapping a number of times which does not exceed the order of the point. That is, if $o(x)$ is the order of the point $x$ and $m(x)$ is the number of points in $f^{-1}(x)$, then $m(x) \leqq o(x)$ for each point for which $o(x)$ is finite. On the other hand, if $\mathcal{X}$ is of dimension $n$, then any continuous mapping of a 1-dimensional compact set onto $\mathscr{X}$,

\footnotetext{
${ }^{1}$ G. Nöbeling, Reguläre Kurven als Bilder der Kreislinie, Fundamenta Mathematicae, vol. 20 (1933), pp. 30-46.

2 The interval may be used instead of the circle if we make $f(0)=f(1)$ and count inverses on $0 \leqq t<1$.
}

Presented to the Society, September 5, 1941; received by the editors October 21, 1941. 